NOTA

Esta versión impresa está abreviada. La versión completa

del artículo está en nuestra edición en Internet.

\title{
Lista y referencias de los crustáceos anfípodos (Amphipoda: Gammaridea) del Atlántico occidental tropical
}

\author{
Manuel Ortíz ${ }^{1}$, Alberto Martín ${ }^{2}$ \& Yusbelly J. Díaz \\ 1 Centro de Investigaciones Marinas, Universidad de La Habana, Calle 16 No 114 e/1ra y 3ra Miramar Playa, Habana, \\ Cuba; ortiztouzet@yahoo.com \\ 2 Laboratorio y Colección de Crustáceos Peracáridos, Departamento de Estudios Ambientales e INTECMAR, Universidad \\ Simón Bolívar, Apartado Postal 89000, Sartenejas-Baruta, Venezuela; amartinz@usb.ve, yusdiaz@usb.ve
}

Recibido 09-V-2001. Corregido 25-I-2007. Aceptado 28-II-2007.

\begin{abstract}
Checklist and references of the Amphipod Crustaceans (Gammaridea) from tropical western Atlantic. The published records of Amphipoda Gammaridea of the tropical western Atlantic region were reviewed and compiled in a checklist. A total of 478 species is recorded (52 families, 199 genera). This adds 20 families and 253 species to the last published list. The families are Ampeliscidae, Amphilochidae, Amphitoidae, Anamixidae, Aoridae, Argissidae, Aristiidae, Bateidae, Biancolinidae, Bogidiellidae, Cheluridae, Colomastigidae, Corophiidae, Cyphocarididae, Cyproideidae, Dexaminidae, Endevouridae, Epimeriidae, Eusiridae, Gammaridae, Hadziidae, Haustoriidae, Hyalellidae, Hyalidae, Hyperiopsidae, Iphimediidae, Isaeidae, Ischyroceridae, Leucothoidae, Liljeborgiidae, Lysianassidae, Megaluropidae, Melitidae, Melphidippidae, Ochlesidae, Oedicerotidae, Pardaliscidae, Phliantidae, Phoxocephalidae, Platyischnopidae, Pleustidae, Podoceridae, Pontoporeiidae, Scopelocheiridae, Sebidae, Stegocephalidae, Stenothoidae, Synopiidae, Talitridae, Uristidae, and Vitjazianidae. Rev. Biol. Trop. 55 (2): 479-498. Epub 2007 June, 29.
\end{abstract}

Key words: diversity, Amphipoda, Gammaridea, Tropical Western Atlantic.

La publicación de listas de especies marinas de diversas regiones geográficas del mundo son una herramienta básica, ya que sirven para determinar la biodiversidad presente en las diferentes localidades, informando sobre la complejidad de las comunidades biológicas y de la disponibilidad de recursos vivos, ayudando a reconocer y delimitar la extensión de zonas protegidas o por proteger, y a determinar el impacto potencial antropogénico (Hendrickx 1995, Escobar-Briones y Winfield 2003).

El Subphylum Crustacea, es el único grupo extenso y diverso de artrópodos básicamente marinos, aunque también abundan las especies dulceacuícolas (Bânârescu 1990, 1995), los cuales han colonizado todo tipo de hábitats, desde los polos hasta el ecuador (Meglitsch y Schram 1991, De la Fuente 1994). Existen además, unos cuantos grupos semiterrestres y terrestres (Cloudsley-Thompson 1988), pero en general estos últimos no han tenido una considerable adaptación evolutiva para la existencia en la tierra.

Dentro de los crustáceos, la Clase Malacostraca es la más numerosa ya que contiene casi $75 \%$ de las especies conocidas de estos organismos, siendo los peracáridos aproximadamente $40 \%$ de todos los malacostracos (Hayward y Ryland 1990, 1995, Meglitsch y Schram 1991, De la Fuente 1994), al cual pertenecen los anfípodos, el segundo orden tanto en número de especies (más de 7 000) como en importancia (Barnard y Karaman 1991, BellanSantini 1999).

Hasta 1979 se conocía un total de 255 especies de anfípodos en el Atlántico occidental tropical, de las cuales 225 pertenecían al suborden Gammaridea (Ortíz 1979a). 
Posteriormente, Camp et al. (1998), informan $216 \mathrm{spp}$ para Florida; mientras que EscobarBriones y Winfield (2003) informan 98 especies presentes en el Golfo de México. Desde entonces, se han citado o descrito muchas especies, lo que unido a las modificaciones taxonómicas del grupo propuestas por diferentes investigadores (Barnard y Barnard 1983, 1990a, b, c, d, Barnard y Karaman 1991, Bousfield y Hoover 1997, Krapp-Schickel y Ruffo 2000), complican la determinación de cualquier especie del grupo. Por otra parte, también resulta muy difícil revisar la creciente literatura especializada al respecto, dispersa en numerosas publicaciones.

Actualmente, el orden de los anfípodos gamarideos del Atlántico occidental tropical puede ser considerado como un grupo de invertebrados bien estudiados, gracias al esfuerzo de un conjunto de especialistas del área unidos a algunos otros europeos, quienes han venido publicando numerosos nuevos taxa en los últimos 60 años, con la excepción de la región sur oriental del Caribe que ha sido poco estudiada.

El objetivo de este estudio, fue la compilación de información sobre la diversidad de anfípodos del Atlántico occidental tropical, suministrando una lista que incluya todas las especies, generos y familias de anfípodos gamarideos, que han sido mencionadas en la literatura para el área hasta el año 2006.

\section{MATERIALES Y MÉTODOS}

Este trabajo incluye todas las especies de anfípodos gamarideos, marinos, dulceacuícolas, anquialinos, estuarinos, troglobios y semiterrestres, que han sido citados previamente para el Atlántico occidental tropical (Bermudas, Florida, Bahamas, Golfo de México, Barbados y Mar Caribe), hasta el año 2006 inclusive; excluyendo los registros dudosos y comunicaciones personales. En el caso de los anquialinos, dulceacuícolas y algunos estuarinos se coloca como distribución el país donde ha sido informada la especie, a diferencia de los marinos donde se presenta la región donde se ha informado y no se especifica el país, para los usuarios que necesiten saber la distribución exacta se recomienda ir a las fuentes originales.

Las especies han sido validadas según Barnard y Barnard (1983), Barnard y Karaman (1991), Holsinger (1992), Thomas y Barnard (1992), Ortíz et al. (1992, 1993), Baldinger y Gable (1994), Ortíz y Lalana (1994a, b), Conlan (1995), LeCroy (1995), Ortíz y Pérez (1995), Thomas y Ortíz (1995), Ortíz y Lalana (1996), Bellan-Santini (1997), Lowry y Stoddart (1997), Ortíz y Lemaitre (1997), Thomas (1997), Martín et al. (2000a, b), Ortíz et al. (2000a, b), Krapp-Schickel y Ruffo (2000), Berge et al. (2001), Díaz y Martín (2001b), Krapp-Schickel y Ruffo (2001), EscobarBriones et al. (2002).

Se ha decidido excluir de este trabajo el término de superfamilia siguiendo la clasificación de Martin y Davis (2001), sin embargo, se ha especificado a qué familia pertenecen según las referencias más nuevas, de manera que se ayude al investigador, debido a las variaciones que ha tenido la taxonomía de estos grupos en los últimos años.

\section{RESULTADOS}

Hasta el presente se han informado 478 especies de anfípodos gamarideos para el Atlántico occidental tropical, distribuidos en 52 familias y 199 géneros. Seis familias son las más diversas en número de especies, en orden decreciente: Melitidae (92 especies, 33 géneros), Aoridae (37 especies, 11 géneros), Lysianassidae (27 especies, 20 géneros), Ampeliscidae (25 especies, cuatro géneros), Corophiidae (19 especies, ocho géneros) e Isaeidae (18 especies, cinco géneros). Estas familias totalizan el $45.61 \%$ del total de la fauna de especies de anfípodos de la región del Atlántico occidental tropical.

De las especies listadas, 390 son netamente marinas (81.93\%), 24 troglobias $(5.04 \%), 18$ semiterrestres (3.78\%), 22 anquialinas (4.62 $\%), 12$ dulceacuícolas $(2.52 \%)$ y 10 estuarinas $(2.10 \%)$. 
Por otra parte, las familias Biancolinidae, Cyphocaridae, Cyproideidae, Endevouridae, Epimeridae, Hyalellidae, Hyperiopsidae, Melphidippidae, Ochlesidae, Phliantidae, Pontoporeidae, Scopelocheiridae, Sebidae, Stegocephalidae y Vitzajanidae son las que poseen el menor número de géneros y de especies en comparación con el resto.
Cabe destacar a la familia Colomastigidae, que posee un solo género (Colomastix) conteniendo 12 especies. Asimismo, los géneros más diversos, en número de especies, de todos los informados para el Atlántico occidental tropical son: Ampelisca que posee 21 especies, Bemlos con 22 especies, Metaniphargus con 20 y finalmente Quadrimaera y Elasmopus con 10 especies cada uno.

\section{Listado sistemático de las especies \\ Suborden Gammaridae Latreille, 1803}

Familia Ampeliscidae Costa, 1857

Ampelisca abdita Mills, 1964

Ampelisca abyssicola Stebbing, 1888

Ampelisca agassizi (Judd, 1896)

Ampelisca bicarinata Goeke \& Heard, 1983

Ampelisca brevisimulata Barnard, 1954

Ampelisca burkei Barnard \& Thomas, 1989

Ampelisca cristata cristata Holmes, 1908

Ampelisca cristata microdentata Barnard, 1954

Ampelisca cristoides Barnard, 1954

Ampelisca holmesi Pearse, 1908

Ampelisca lobata Holmes, 1908

Ampelisca parapacifica Goeke \& Heard, 1984

Ampelisca parapanamensis Barnard, 1954

Ampelisca paria Barnard \& Agard, 1986

Ampelisca pugetica Stimpson, 1864

Ampelisca romigi Barnard, 1954

Ampelisca schellenbergi Shoemaker, 1933

Ampelisca spinipes Boeck, 1861

Ampelisca vadorum Mills, 1963

Ampelisca venetiensis Shoemaker, 1916

Ampelisca verrilli Mills, 1967

Byblis cubensis (Ortíz \& Gómez, 1979)

Byblis serrata Smith, 1873

Byblisoides blasensis Barnard, 1964

Haploops setosa Boeck, 1871

Familia Amphilochidae Boeck, 1871

Amphilochus ascidicola Martín, Ortíz \& Atienza, 2001

Amphilochus casahoya McKinney, 1978

Amphilochus delacaya McKinney, 1978

Amphilochus neapolitanus Della Valle, 1893

Amphilochus pillaii Barnard \& Thomas, 1983

Gitana dominica Thomas \& Barnard, 1990

Gitanopsis laguna McKinney, 1978
Gitanopsis petulans Karaman, 1980

Gitanopsis templadoi Ortíz \& Lalana, 1995

Gitanopsis tortugae Shoemaker, 1933

Familia Ampithoidae Stebbing, 1899

Ampithoe hirsutimanus Ortíz \& Lemaitre, 1997

Ampithoe longimana Smith, 1873

Ampithoe marcuzzii Ruffo, 1954

Ampithoe pollex Kunkel, 1910

Ampithoe ramondi Audouin, 1826

Ampithoe valida Smith, 1873

Cymadusa compta (Smith, 1873)

Cymadusa filosa Savigny, 1816

Perampithoe femorata (Kroyer, 1845)

Pseudoamphithoides bacescui Ortíz, 1976

Pseudoamphitoides incurvaria (Just, 1977)

Sunamphitoe pelagica Milne Edwards, 1830

Familia Anamixidae Stebbing, 1897

Anamixis cavatura Thomas, 1997

Anamixis cavatura morfo Leucothoides Thomas, 1997

Anamixis hanseni Stebbing, 1897

Anamixis vanga Thomas, 1997

Nepanamixis dianthus Thomas, 1997

Familia Aoridae Walker, 1908

Amphideutopus dolichocephalus Myers, 1968

Bemlos barnardi (Ortíz \& Nazábal, 1988)

Bemlos brunneomaculatus brunneomaculatus

(Myers, 1977)

Bemlos brunneomaculatus mackinneyi (Myers, 1978)

Bemlos dentischium (Myers, 1977)

Bemlos foresti (Mateus \& Mateus, 1966)

Bemlos habanensis Ortíz, 1980

Bemlos kunkelae (Myers, 1977) 
Bemlos longicornis (Myers, 1978)

Bemlos mackinneyi (Myers, 1978)

Bemlos macromanus Shoemaker, 1925

Bemlos mayensis Ortíz \& Nazábal, 1984

Bemlos minimus (Myers, 1977)

Bemlos sanmartini Ortíz, Lalana \& López, 1992

Bemlos scolosternum (Ortíz \& Lemaitre, 1997)

Bemlos setosus (Myers, 1978)

Bemlos spinicarpus inermis (Myers, 1979)

Bemlos spinicarpus spinicarpus (Pearse, 1912)

Bemlos tempus (Myers, 1979)

Bemlos tigrinus (Myers, 1979)

Bemlos unicornis (Bynum \& Fox, 1977)

Bemlos unifasciatus unifasciatus (Myers, 1977)

Bemlos unifasciatus reductus Myers, 1979

Globosolembos smithi (Holmes, 1905)

Lembos websteri Bate, 1857

Leptocheirus plumulosus Shoemaker, 1932

Leptocheirus rhizophorae Ortíz \& Lalana, 1980

Liocuna caeca Myers, 1981

Paramicrodeutopus myersi (Bynum \& Fox, 1977)

Plesiolembos ovalipes (Myers, 1979)

Plesiolembos rectangulatus (Myers, 1977)

Pseudomegamphopus excavatus (Myers, 1968)

Rudilemboides heardi Ortíz \& Lalana, 1996

Rudilemboides naglei Bousfield, 1973

Unciola dissimilis Shoemaker, 1945

Unciola serrata Shoemaker, 1945

Unciola spicata Shoemaker, 1945

Familia Argissidae Walker, 1904

Argissa hamatipes (Norman, 1869)

Familia Aristiidae Lowry \& Stoddart, 1997

Aristias captiva Lowry \& Stoddart, 1997

Boca campi Lowry \& Stoddart, 1997

Boca elvae Lowry \& Stoddart, 1997

Boca megachela Lowry \& Stoddart, 1997

Miramarassa sanchezi Ortíz, Lalana \& Lio, 1999

Familia Bateidae Stebbing, 1906

Batea bousfieldi (Ortíz, 1991)

Batea campi (Ortíz, 1991)

Batea carinata (Shoemaker, 1926)

Batea catharinensis Müller, 1865

Batea cuspidata (Shoemaker, 1926)

Batea schotti Ortíz \& Lemaitre, 1997
Familia Biancolinidae Barnard, 1972

Biancolina brassicacephala Lowry, 1974

Biancolina lowryi Ortíz \& Lalana, 1996

Familia Bogidiellidae Hertzog, 1936

Actogidiella cultrifera Stock, 1981

Bermudagidiella bermudensis (Stock, Sket \& Iliffe, 1987)

Bogidiella bredini Shoemaker, 1959

Bogidiella perla Stock, 1981

Bogidiella virginalis Stock, 1981

Marigidiella crassipes Stock, 1981

Familia Cheluridae Allman, 1847

Chelura terebrans Philippi, 1839

Tropichelura gomezi Ortíz, 1976

Tropichelura insulae (Calman, 1910)

Familia Colomastigidae Stebbing, 1899

Colomastix bousfieldi LeCroy, 1995

Colomastix camura LeCroy, 1995

Colomastix cornuticauda LeCroy, 1995

Colomastix denticornis LeCroy, 1995

Colomastix falcirama LeCroy, 1995

Colomastix gibbosa LeCroy, 1995

Colomastix halichondriae Bousfield, 1973

Colomastix heardi LeCroy, 1995

Colomastix irciniae LeCroy, 1995

Colomastix janiceae Heard \& Perlmutter, 1977

Colomastix pusilla Grube, 1861

Colomastix tridentata LeCroy, 1995

Familia Corophiidae Dana, 1849

Americorophium aquafuscum (Heard \& Sikora, 1972)

Americorophium ellisi (Shoemaker, 1943)

Americorophium rioplatense Giambiagi, 1929

Apocorophium acutum (Chevreux, 1908)

Apocorophium lacustre (Vanhöffen, 1911)

Apocorophium louisianum (Shoemaker, 1934)

Apocorophium simile (Shoemaker, 1934)

Caribboecetes barbadensis Just, 1984

Caribboecetes crassicornis Just, 1984

Caribboecetes intermedius Just, 1984

Caribboecetes justi Ortíz \& Lemaitre, 1997

Caribboecetes pterycornis Just, 1984

Crassicorophium bonelli (H. Milne Edwards, 1830)

Grandidierella bonnieroides Stephensen, 1948

Laticorophium cf. baconi (Shoemaker, 1934) 
Monocorophium acherusicum (Costa, 1851)

Monocorophium insidiosum (Crawford, 1937)

Monocorophium tuberculatum (Shoemaker, 1934)

Pedicorophium laminosum (Pearse, 1912)

Familia Cyphocarididae Lowry y Stoddart, 1997

Cyphocaris johnsoni Shoemaker, 1934

Cyphocaris tunicola Lowry \& Stoddart, 1997

Familia Cyproideidae Barnard, 1974

Hoplopheonoides obesa Shoemaker, 1956

Hoplophoenoides shoemakeri Ortíz, Lalana \& SánchezDíaz, 2000

Familia Dexaminidae Leach, 1814

Atylus minikoi (Walker, 1905)

Lepechinella aberrantis (Barnard, 1962)

Lepechinella raua Barnard, 1973

Familia Endevouridae Lowry \& Stoddart, 1997

Ensayara entrichoma Gable \& Lazo-Wasem, 1990

Ensayara jumane Barnard \& Thomas, 1990

Familia Epimeriidae Boeck, 1871

Epimeria longispinosa Barnard, 1916

Epimeria obtusa Watling, 1981

Familia Eusiridae Stebbing, 1888

Eusirogenes adad Barnard, 1964

Eusiroides monoculoides (Haswell, 1879)

Eusiroides yucatanensis McKinney, 1980

Nasageneia bacescui Ortíz \& Lalana, 1994

Nasageneia comisariensis Ortíz \& Lemaitre, 1997

Nasageneia yucatanensis Ledoyer, 1986

Pontogeneia bartschi Shoemaker, 1948

Rachotropis lobata Shoemaker, 1934

Rachotropis portoricana Barnard, 1964

Sennaia bidactyla Bellan-Santini, 1997

Tethygeneia longleyi (Shoemaker, 1933)

Familia Gammaridae Latreille, 1802

Gammarus fasciatus Say, 1818

Gammarus minus Say, 1818

Gammarus mucronatus (Say, 1818)

Gammarus palustris Bousfield, 1969

Gammarus tigrinus Sexton, 1939

Marinogammarus finmarchicus (Dahl, 1938)
Familia Hadziidae Karaman, 1943

Bahadzia bozanici Holsinger, 1992

Bahadzia jaraquensis Jaume \& Wagner, 1998

Bahadzia latipalpus Stock, 1985

Bahadzia obliqua Stock, 1986

Bahadzia setimana Stock, 1986

Bahadzia setodactylus Holsinger, 1992

Bahadzia stocki Holsinger \& Yager, 1985

Bahadzia williamsi Holsinger \& Yager, 1985

Bahadzia yagerae Ortíz \& Pérez, 1995

Protohadzia schoenerae (Fox, 1973)

Familia Haustoriidae Stebbing, 1906

Acanthohaustorius bousfieldi Frame, 1980

Acanthohaustorius intermedius Bousfield, 1965

Acanthohaustorius millsi Bousfield, 1965

Acanthohastorius pansus Thomas \& Barnard, 1984

Acanthohaustorius shoemakeri Bousfield, 1965

Acanthohaustorius uncinus Foster, 1989

Haustorius arenarius (Slabber, 1769)

Haustorius canadensis Bousfield, 1962

Haustorius jayneae Foster \& LeCroy, 1991

Haustorius mexicanus Ortíz, Chazaro-Olvera \& Winfield, 2001

Lepidactylus dysticus Say, 1818

Lepidactylus triarticulatus Robertson \& Shelton, 1980

Neohaustorius schmitzi Bousfield, 1965

Parahaustorius holmesi Bousfield, 1965

Parahaustorius longimerus Bousfield, 1965

Parahaustorius obliquus Robertson \& Shelton, 1978

Pseudohaustorius americanus (Pearse, 1908)

Pseudohaustorius caroliniensis Bousfield, 1965

Protohaustorius bousfieldi Robertson \& Shelton, 1978

Protohaustorius deichmannae Bousfield, 1965

Protohaustorius wigleyi Bousfield, 1965

Familia Hyalellidae Bulycheva, 1957

Hyalella anophthalma Ruffo, 1957

Hyalella azteca Saussure, 1858

Hyalella faxoni Stebbing, 1903

Parhyalella batesoni Kunkel, 1910

Parhyalella whelpleyi Shoemaker, 1933

Familia Hyalidae Bulycheva, 1957

Hyale diplodactyla Stebbing, 1899

Hyale macrodactyla Stebbing, 1899

Hyale media (Dana, 1853) 
Hyale perieri (Lucas, 1846)

Hyale pygmaea Ruffo, 1950

Parhyale fascigera Stebbing, 1897

Parhyale hawaiensis (Dana, 1853)

Parhyale inyacka Barnard, 1916

Familia Hyperiopsidae Bovallius, 1886

Parargissa galatheae Barnard, 1961

Familia Iphimediidae Boeck, 1871

Iphimedia zora Thomas \& Barnard, 1991

Familia Isaeidae Dana, 1853

Audulla chelifera Chevreux, 1901

Chevalia aviculae Walker, 1904

Chevalia carpenteri Barnard \& Thomas, 1987

Chevalia mexicana Pearse, 1913

Gammaropsis arawakia Thomas y Barnard, 1989

Gammaropsis atlantica Stebbing, 1888

Gammaropsis kaumaka Barnard, 1970

Gammaropsis togoensis (Schellenberg, 1925)

Gammaropsis sutherlandi Nelson, 1980

Microprotopus raneyi Wigley, 1966

Microprotopus shoemakeri Lowry, 1972

Photis dentata Shoemaker, 1945

Photis longicaudata (Bate \& Westwood, 1862)

Photis macromanus McKinney, Kalke \& Holland, 1978

Photis melanica (McKinney, 1980)

Photis pugnator Shoemaker, 1945

Photis reinhardi Kroyer, 1842

Photis trapherus Thomas \& Barnard, 1991

Familia Ischyroceridae Stebbing, 1899

Cerapus benthophilus Thomas \& Heard, 1979

Cerapus cudjoe Lowry \& Thomas, 1991

Cerapus tubularis Say, 1817

Cerapus thomasi Ortíz \& Lemaitre, 1997

Corocubanus guitarti Ortíz \& Nazábal, 1984

Ericthonius brasiliensis (Dana, 1853)

Ericthonius rubricornis (Stimpson, 1853)

Ischyrocerus anguipes Kroyer, 1838

Jassa falcata (Montagu, 1808)

Microjassa bahamensis Conlan, 1995

Microjassa floridensis Conlan, 1995

Microjassa tetradonta Conlan, 1995

Neoischyrocerus vidali Ortiz \& Lalana, 2002
Familia Leucothoidae Dana, 1852

Leucothoe ashleyae Thomas \& Klebba, 2006

Leucothoe ayrtonia Bellan-Santini, 1997

Leucothoe kensleyi Thomas \& Klebba, 2006

Leucothoe laurensi Thomas \& Ortíz, 1995

Leucothoe panpulco Barnard, 1961

Leucothoe spinicarpa (Abildgaard, 1789)

Familia Liljeborgiidae Stebbing, 1899

Idunella sketi Karaman, 1980

Liljeborgia bousfieldi McKinney, 1979

Liljeborgia dellavallei Stebbing, 1906

Liljeborgia pallida (Bate, 1857)

Listriella andresi Martín, Ortíz \& Atienza, 2000

Listriella bahia McKinney, 1979

Listriella barnardi Wigley, 1966

Listriella carinata McKinney, 1979

Listriella clymenellae Mills, 1962

Listriella kensleyi Ortíz \& Lalana, 1996

Listriella quintana McKinney, 1979

Familia Lysianassidae Dana, 1849

Aruga holmesi Barnard, 1955

Bonassa bonairensis (Stephensen, 1933)

Cebocaris grutesca Barnard, 1964

Concarnes concavus (Shoemaker, 1933)

Dissiminassa homosassa Lowry \& Stoddart, 1997

Eclecticus eclecticus Lowry \& Stoddart, 1997

Eurythenes gryllus (Lichtenstein, 1822)

Eurythenes obesus (Chevreux, 1905)

Falcanassa falcata (Stephensen, 1933)

Hippomedon pensacola Lowry \& Stoddart, 1997

Lepiduristes lepidus (Barnard, 1964)

Lysianopsis alba Holmes, 1903

Lysianopsis hummelincki (Stephensen, 1933)

Lysianopsis ozona Lowry \& Stoddart, 1997

Lysianopsis subantarctica (Schellenberg, 1931)

Orchomene stocki Bellan-Santini, 1990

Orchomenella perdido Lowry \& Stoddart, 1997

Orchomenella thomasi Lowry \& Stoddart, 1997

Orenoquia serrata Bellan-Santini, 1997

Ottenwalderia kymbalion Jaume \& Wagner, 1998

Paracentromedon carabicus Barnard, 1964

Rimakoroga floridiana Lowry \& Stoddart, 1997

Shoemakerella cubensis (Stebbing, 1897)

Shoemakerella lowryi Gable \& Lazo-Wasem, 1990 
Socarnopsis catacumba (Clark \& Barnard, 1985)

Tryphosella apalachicola Lowry \& Stoddart, 1997

Tryphosella uristesi Bellan-Santini, 1997

Familia Megaluropidae Thomas \& Barnard, 1986

Gibberosus myersi (McKinney, 1980)

Resupinus spinicaudatus Thomas \& Barnard, 1986

Familia Melitidae Bousfield, 1973

Alloweckelia gurneei Holsinger \& Peck, 1968

Anamaera hixoni Thomas \& Barnard, 1985

Anelasmopus kraui Oliveira, 1953

Apoweckelia serrata Stock, 1985

Ceradocus rubromaculatus (Stimpson, 1856)

Ceradocus sheardi Shoemaker, 1948

Ceradocus shoemakeri Fox, 1973

Confodiopisa caesicola (Stock, 1980)

Confodiopisa scopulorum (Stock, 1983)

Crangoweckelia mixta Stock, 1985

Crangoweckelia spinicauda Stock, 1985

Dulichiella appendiculata (Say, 1818)

Dumosus atari Thomas \& Barnard, 1985

Elasmopus balkomanus Thomas \& Barnard, 1988

Elasmopus bampo Barnard, 1979

Elasmopus brasiliensis (Dana, 1853)

Elasmopus lemaitre Ortíz \& Lalana, 1994

Elasmopus levis (Smith, 1873)

Elasmopus pectenicrus (Bate, 1862)

Elasmopus pocillimanus (Bate, 1862)

Elasmopus rapax Costa, 1853

Elasmopus spinidactylus Chevreux, 1907

Elasmopus thomasi Ortíz \& Lalana, 1994

Eriopisa incisa McKinney, Kalke \& Holland, 1978

Eriopisa mochimae Van der Ham \& Vonk, 2003

Gammarella carausui (Ortíz, 1976)

Gammarella curvata (Vonk, 1989)

Gammarella muelleri (Ortíz, 1976)

Jerbarnia americana Watling, 1981

Maera caroliniana Bynum \& Fox, 1977

Maera rathbunae Pearse, 1908

Maera tinkerensis Kunkel, 1910

Maera williamsi Bynun \& Fox, 1977

Maeracoota galani Krapp-Schickel \& Ruffo, 2001

Mayaweckelia cenoticola Holsinger, 1977

Mayaweckelia yucatanensis Holsinger, 1977

Melita dentata Kroyer, 1842

Melita elongata Sheridan, 1979

Melita intermedia Sheridan, 1980
Melita leiotelson Vonk, 1988

Melita longisetosa Sheridan, 1980

Melita nitida Smith, 1873

Melita persona Karaman, 1981

Melita stocki Karaman, 1987

Metaniphargus anchihalinus Stock, 1983

Metaniphargus beattyi Shoemaker, 1942

Metaniphargus bousfieldi Stock, 1977

Metaniphargus chaetodactylus Stock, 1985

Metaniphargus craterensis Stock, 1983

Metaniphargus crenatus Stock, 1985

Metaniphargus curasavicus curasavicus Stephensen, 1933

Metaniphargus haitianus Stock, 1985

Metaniphargus hyporheicus Stock, 1983

Metaniphargus jamaicae (Holsinger, 1974)

Metaniphargus juberthiei Stock, 1985

Metaniphargus longipalpus Stock, 1985

Metaniphargus longipes christophorensis Stock, 1977

Metaniphargus longipes longipes Stock, 1977

Metaniphargus nicholsoni Shoemaker, 1959

Metaniphargus palpator Stock, 1977

Metaniphargus pedunculatus Stock, 1985

Metaniphargus plumicauda Stock, 1985

Metaniphargus sabulonis Vonk, 1991

Metaniphargus venezolanus Stock \& Botosaneanu, 1983

Netamelita barnardi McKinney, Kalke \& Holland, 1978

Netamelita brocha Thomas \& Barnard, 1991

Netamelita tabaci Thomas \& Barnard, 1991

Paraweckelia silvai Shoemaker, 1959

Pintoweckelia grandis Stock, 1985

Psammogammarus bluefieldensis Ortíz, Lalana \& Beltran, 1993

Psammogammarus longidactylus Vonk \& Stock, 1987

Psammomelita uncinata Vonk, 1988

Quadrimaera ariel Ruffo, Krapp \& Gable, 2000

Quadrimaera inaequipes (Costa, 1851)

Quadrimaera caliban (Ruffo, Krapp \& Gable, 2000)

Quadrimaera ceres Ruffo, Krapp \& Gable, 2000

Quadrimaera cristianae Krapp-Schickel \& Ruffo, 2000

Quadrimaera quadrimana (Dana, 1853)

Quadrimaera miranda (Ruffo, Krapp \& Gable, 2000)

Quadrimaera pacifica (Schellenberg, 1938)

Quadrimaera pieteri Krapp-Schichel \& Ruffo, 2000

Quadrimaera sarae Krapp-Schickel \& Ruffo, 2000

Quadrivisio lutzi (Shoemaker, 1933)

Radaweckelia brevicauda Stock, 1985

Saliweckelia emarginata Stock, 1977

Saliweckelia holsingeri Stock, 1977 
Spathiopus looensis Thomas \& Barnard, 1985

Thalassostygius exiguus Vonk, 1990

Tuluweckelia cernua Holsinger, 1990

Victoriopisa guanarocana Ortíz \& Lalana, 1989

Weckelia caeca (Weckel, 1907)

Zombiweckelia parvipalpus Stock, 1985

\section{Familia Melphidippidae Stebbing, 1899}

Hornellia (Metaceradocus) atlanticus Thomas \& Barnard, 1986

Hornellia (Metaceradocus) tequestae Thomas \& Barnard, 1986

Familia Neomegamphopidae Myers, 1981 Neomegamphopus heardi Barnard \& Thomas, 1987 Neomegamphopus hiatus Barnard \& Thomas, 1987 Neomegamphopus kalanii Barnard \& Thomas, 1987 Neomegamphopus roosevelti Shoemaker, 1942

Familia Ochlesidae Stebbing, 1910 Curidia debrogania Thomas, 1983

Familia Oedicerotidae Lilljeborg, 1865 Americhelidium americanum (Bousfield, 1973) Ameroculodes edwardsi (Holmes, 1903) Deflexilodes intermedius (Shoemaker, 1930) Hartmanodes nyei (Shoemaker, 1933) Oediceroides synparis Barnard, 1964 Perioculodes cerasinus Thomas \& Barnard, 1985 Westwoodilla longimana Shoemaker, 1934

Familia Pardaliscidae Boeck, 1871

Parpano cebus Barnard, 1964

Parpano composturus Barnard, 1964

Spelaeonicippe provo Stock \& Vermeulen, 1982

Familia Phliantidae Stebbing, 1899

Pariphinotus seclusus (Shoemaker, 1933)

Pariphinotus seticoxa (Ortíz, 1976)

Familia Phoxocephalidae Sars, 1895

Cocoharpinia iliffei Karaman, 1980

Eobrolgus spinosus (Holmes, 1903)

Harpinia excavata Chevreux, 1887

Harpinia intermedia Bellan-Santini, 1997

Heterophoxus oculatus (Holmes, 1908)

Metharpinia floridana (Shoemaker, 1933)

Microphoxus minimus Barnard, 1960
Proharpinia barbada Bellan-Santini, 1997

Rhepoxynius epistomus (Shoemaker, 1938)

Rhepoxynius hudsoni Barnard \& Barnard, 1982

Familia Platyischnopidae Barnard \& Drummond, 1979

Eudevenopus honduranus Thomas \& Barnard, 1983

Eudevenopus metagracilis (Barnard, 1964)

Tiburonella morrocoyensis Ortíz, Martín \& Atienza, 2000

Tiburonella viscana (Barnard, 1964)

Familia Pleustidae Buchholz, 1874

Parapleustes aestuarius Watling \& Maurer, 1973

Stenopleustes gracilis (Holmes, 1903)

Familia Podoceridae Leach, 1814

Podobothrus bermudensis Barnard \& Clark, 1985

Podocerus brasiliensis (Dana, 1853)

Podocerus cristatus (Thomson, 1879)

Podocerus chelonophilus (Chevreux \& de Guerne, 1888)

Podocerus kleidus Thomas \& Barnard, 1992

Podocerus lazowasemi Baldinger \& Gable, 1994

Podocerus tachyrheo Baldinger \& Gable, 1994

Familia Pontoporeiidae Dana, 1853

Bathyporeia parkeri Bousfield, 1973

Familia Scopelocheiridae Lowry \& Stoddart, 1997 Aroui americana Lowry \& Stoddart, 1997

Familia Sebidae Walker, 1908

Seba aloe Karaman, 1971

Seba robusta Ortíz \& Lemaitre, 1997

Seba tropica McKinney, 1980

Familia Stegocephalidae Dana, 1855

Stegocephaloides calypsonis Berge, Vader \& Galan, 2001

Familia Stenothoidae Boeck, 1871

Metopella nasuta Boeck, 1861

Parametopella cypris (Holmes, 1903)

Parametopella inquilina Watling, 1976

Parametopella texensis McKinney, Kalke \& Holland, 1978

Stenothoe gallensis Walker, 1904

Stenothoe georgiana Bynum \& Fox, 1977

Stenothoe minuta Holmes, 1903

Stenothoe symbiotica Shoemaker, 1956 
Familia Synopiidae Dana, 1853

Bruzelia pericu Barnard, 1972

Bruzeliopsis turba Barnard, 1964

Garosyrrhoe bigarra (Barnard, 1962)

Garosyrrhoe luquei Ortíz \& Veledo, 1985

Ileraustroe torpens (Barnard, 1962)

Latacunga comanita Barnard, 1972

Metatiron bellairsi (Just, 1981)

Metatiron triocellatus (Goeke, 1982)

Metatiron tropakis (Barnard, 1972)

Synopia scheeleana Bovallius, 1886

Synopia ultramarina Dana, 1853

Syrrhoe papyracea Stebbing, 1888

Familia Talitridae Rafinesque, 1815

Americorchestia barbarae Bousfield, 1991

Americorchestia heardi Bousfield, 1991

Americorchestia longicornis (Say, 1818)

Americorchestia megalophthalma (Bate, 1862)

Americorchestia salomani Bousfield, 1991

Chelorchestia costaricana Stebbing, 1906

Chelorchestia forceps Smith \& Heard, 2001

\section{DISCUSIÓN}

Ortíz (1979) informa 225 especies de anfípodos gamarideos marinos para el Atlántico occidental tropical, mientras Barnard y Karaman (1991) señalan 210. Sin embargo, en estos últimos años su número ha aumentado significativamente hasta las 478 especies aquí informadas. Desde la publicación de 1979 hasta el año 2006 se han añadido a la lista 253 especies, de las cuales 66 han sido nuevas descripciones para la ciencia y 68 nuevos informes para la región. Sin embargo, esta lista no pretende ser la definitiva, dado que en muchos países de la región, se está comenzando a trabajar con estos organismos, lo cual podría aportar nuevos informes para el área, así como nuevos géneros y especies para la ciencia.

El Golfo de México, las costas de Florida, Cuba, Venezuela y Colombia han sido los países donde más se ha estudiado la fauna de los anfípodos, pero faltan trabajos intensivos en el resto de los países. De los anfípodos Gammaridea, han sido estudiadas en su mayoría las especies marinas, por ser las más
Floresorchestia guadalupensis Ciavatti, 1989

Orchestia grillus (Bosc, 1802)

Platorchestia platensis (Kroyer, 1845)

Talorchestia fritzi Stebbing, 1903

Talorchestia longicornis (Say, 1818)

Talorchestia marcuzzi Ruffo, 1950

Talorchestia margaritae Stephensen, 1947

Thetorchestia antillensis Bousfield, 1984

Thetorchestia karukerae Ciavatti, 1989

Thetorchestia sulensoni (Stebbing, 1899)

Uhlorchestia spartinophila Bousfield \& Heard, 1986

Uhlorchestia uhleri (Shoemaker, 1930)

Familia Uristidae Hurley, 1963

Stephonyx biscayensis (Chevreux, 1908)

Stephonyx carinatus Bellan-Santini, 1997

Stephonyx incertus Bellan-Santini, 1997

Familia Vitjazianidae Barnard \& Vinogradov, 1955

Vemana compressa Barnard, 1964

Vemana lizata Barnard, 1964

abundantes, quedando rezagadas las dulceacuícolas, semiterrestres, terrestres, anquialinas y estuarinas, razón por la cual, los anfípodos gamarideos marinos son los mejores representados en esta lista.

La preferencia de habitat, resulta ser un aspecto poco conocido, aunque existen datos que indican que varias familias ocurren de manera frecuente en cierto tipo de ambientes, como los fondos blandos (Ampeliscidae y Platyischnopidae), mientras que en la gran mayoria, la diversidad de habitat es muy amplia.

En conclusión, los anfípodos del suborden Gammaridea en el Atlántico occidental tropical, han sido bien estudiados en su conjunto; aún cuando por ser un grupo muy diverso puede ser difícil de trabajar cuando no se dispone de la literatura necesaria y actualizada, recomendándose fomentar su estudio en la mayoría de las costas e islas de la región. Por otra parte, la distribución de la mayoría de las especies no se conoce a plenitud y aunque en muchos casos se informa presente en el Mar Caribe, es sólo para un país en particular, dado que todavía no se ha 
encontrado en otros, y esto sea probablemente por un lado debido a la falta de muestreos y por otro lado a la falta de investigadores en el área de la taxonomía de anfípodos. También se hace necesario incluir en los estudios de anfípodos más información sobre su ecología y biología.

\section{AGRADECIMIENTOS}

Agradecemos profundamente a los árbitros $\mathrm{y}$ editores por las detalladas observaciones y sugerencias realizadas, así como a los numerosos autores que gentilmente nos enviaron sus publicaciones para contribuir con esta revisión.

\section{RESUMEN}

Se presenta una lista actualizada de los anfípodos gamarideos del Atlántico occidental tropical, la cual contiene 52 familias y 478 especies. En comparación con trabajos anteriores, el número de familias y especies de esta región se incrementó en 20 y 253, respectivamente. Las familias presentes en la lista son las siguientes: Ampeliscidae, Amphilochidae, Amphitoidae, Anamixidae, Aoridae, Argissidae, Aristiidae, Bateidae, Biancolinidae, Bogidiellidae, Cheluridae, Colomastigidae, Corophiidae, Cyphocarididae, Cyproideidae, Dexaminidae, Endevouridae, Epimeriidae, Eusiridae, Gammaridae, Hadziidae, Haustoriidae, Hyalellidae, Hyalidae, Hyperiopsidae, Iphimediidae, Isaeidae, Ischyroceridae, Leucothoidae, Liljeborgiidae, Lysianassidae, Megaluropidae, Melitidae, Melphidippidae, Neomegamphopidae, Ochlesidae, Oedicerotidae, Pardaliscidae, Phliantidae, Phoxocephalidae, Platyischnopidae, Pleustidae, Podoceridae, Pontoporeiidae, Scopelocheiridae, Sebidae, Stegocephalidae, Stenothoidae, Synopiidae, Talitridae, Uristidae y Vitjazianidae.

Palabras clave: diversidad, Amphipoda, Gammaridea, Atlantico Occidental Tropical

\section{REFERENCIAS}

Atienza, D. 2000. Taxonomía y ecología de los anfípodos asociados a praderas de Thalassia testudinum en el Parque Nacional Morrocoy. Tesis de Licenciatura, Universidad Simón Bolívar, Caracas, Venezuela.
Atienza, D. \& A. Martín. 2001. Tiburonella viscana (Amphipoda: Plastyisnopidae) en las costas de Venezuela. Rev. Biol. Trop. 49: 1270.

Ayala, Y. \& A. Martín. 2003. Ampliación del ámbito de Podocerus kleidus (Amphipoda: Podoceridae) al Mar Caribe. Rev. Biol. Trop. 51: 267.

Baldinger, A.J. \& M.F. Gable. 1994. Two new species of Podocerus Leach (Crustacea, Amphipoda, Podoceridae) from Bermuda. Proc. Biol. Soc. Wash. 107: 707-720.

Bânârescu, P.M. 1990. Zoogeography of fresh waters: General distribution and dispersal of freshwater animals. AULA-Verlag, Wiesbaden, Alemania.

Bânârescu, P.M. 1995. Zoogeography of fresh waters: Distribution and dispersal of freshwater animals in Africa, Pacific areas and South America. AULAVerlag, Wiesbaden, Alemania.

Barnard, J.L. 1954a. Amphipoda of the family Ampeliscidae collected by the Velero III in the Caribbean Sea. Allan Hancock Atlantic Expedition Report 7: 1-13.

Barnard, J.L. 1954b. Amphipoda of the family Ampeliscidae collected in the eastern Pacific ocean by the Velero III and Velero IV. Allan Hancock Pacific Expeditions I: 1-137.

Barnard, J.L. 1955. Gammaridean Amphipoda (Crustacea) in the collections of Bishop Museum. Bull. B.P. Bishop Museum 215: 1-46.

Barnard, J.L. 1959. Generic partition in the amphipod family Cheluridae, marine wood borers. Pacific Naturalist 1: 1-12.

Barnard, J.L. 1960. The amphipoda family Phoxocephalidae in the Eastern Pacific Ocean, with analysis of other species and notes for a revision of the family. Allan Hancock Pacific Expeditions 18: 175-368.

Barnard, J.L. 1961. Gammaridean Amphipoda from depths of 400 to $6000 \mathrm{~m}$. Galathea Report 5: 23-128.

Barnard, J.L. 1962. Benthic marine Amphipoda of Southern California. Familias Aoridae, Photidae, Ischyroceridae, Corophiidae, Podoceridae. Pacific Naturalist 3: 1-72.

Barnard, J.L. 1963. Los anfípodos bentónicos marinos de la costa occidental de Baja California. Rev. Soc. Mex. Hist. Nat. 24: 205-274. 
Barnard, J.L. 1964. Deep-sea Amphipoda (Crustacea) collectec by the R/V "Vema" in the eastern Pacific ocean and the Caribbean and Mediterranean Seas. Bull. Am. Mus. Nat. Hist. 127: 3-46.

Barnard, J.L. 1965. Marine amphipoda of the family Ampithoidae from southern California. Proc. U.S. Nat. Mus. 118: 1-46.

Barnard, J.L. 1966. Benthic amphipoda of Monterey Bay, California. Proc. U.S. Nat. Mus. 119: 1-41.

Barnard, J.L. 1967. New species and records of Pacific Ampeliscidae (Crustacea: Amphipoda). Proc. U.S. Nat. Mus. 121: 1-20.

Barnard, J.L. 1969. The families and genera of marine gammaridean Amphipoda. Bull. U.S. Nat. Mus. 271: $1-535$.

Barnard, J.L. 1970. Sublittoral Gammaridea (Amphipoda) of the Hawaiian Islands. Smith. Contrib. Zool. 34: $1-286$.

Barnard, J.L. 1971. Gammaridean amphipoda from a deep-sea transect off Oregon. Smith. Contrib. Zool 61: $1-86$

Barnard, J.L. 1972. A review of the family Synopiidae (=Tironidae) mainly distributed in the deep sea (Crustacea, Amphipoda). Smith. Contrib. Zool. 124: $1-94$.

Barnard, J.L. 1973. Deep-sea amphipoda of the genus Lepechinella (Crustacea). Smith. Contrib. Zool. 133 $1-31$.

Barnard, J.L. 1980. Revision of Metharpinia and Microphoxus (marine phoxocephalis amphipoda from the Americas). Proc. Biol. Soc. Wash. 93: 104-135.

Barnard, J.L. \& J.B.R. Agard. 1986. A new species of Ampelisca (Crustacea, Amphipoda) from Trinidad. Bull. Mar. Sci. 39: 630-636.

Barnard, J.L. \& C.M. Barnard. 1982. The genus Rhepoxynius (Crustacea: Amphipoda: Phoxocephalidae) in American Seas. Smith. Contrib. Zool. 357: 1-49.

Barnard, J.L. \& C.M. Barnard. 1983. Freshwater Amphipoda of the World I. Evolutionary patterns and II. Handbook and bibliography. Hayfield Associates, Virginia, EEUU.

Barnard, J.L. \& C.M. Barnard. 1990a. Familial index to marine and freshwater Gammaridea (Amphipoda). Division of Crustacea, Nat. Mus. Nat. Hist. Smithsonian Institution, Washington D.C., EEUU.
Barnard, J.L. \& C.M. Barnard. 1990b. Index to marine Gammaridea(Amphipoda)(Except section Gammarida). Division of Crustacea, Nat. Mus. Nat. Hist. Smithsonian Institution, Washington D.C., EEUU.

Barnard, J.L. \& C.M. Barnard. 1990c. Index to freshwater Gammaridea (Amphipoda) (Including marine species of Section Gammarida). Division of Crustacea, Nat. Mus. Nat. Hist. Smithsonian Institution, Washington D.C., EEUU.

Barnard, J.L. \& C.M. Barnard. 1990d. Geographic index to marine Gammaridea (Amphipoda). Division of Crustacea, Nat. Mus. Nat. Hist. Smithsonian Institution, Washington D.C., EEUU.

Barnard, J.L. \& J. Clark. 1985. A new sea-cave amphipod from Bermuda (Dulichiidae). Proc. Biol. Soc. Wash. 98: 1048-1053.

Barnard, J.L. \& G. Karaman. 1991. The families and genera of Marine gammaridean Amphipoda (except marine gammaroids). Rec. Aust. Mus. 13: 1- 866.

Barnard, J.L. \& J.D. Thomas. 1983. A new species of Amphilochus from the gorgonain Pterogorgia anceps in the Caribbean Sea. Festschriff for Dr. Pillai. Selected Papers on Crustacea: 179-187.

Barnard, J.L. \& J.D. Thomas. 1987a. The Indo-pacific Audulla chelifera reported from the Caribbean Sea (Crustacea, Amphipoda). Proc. Biol. Soc. Wash. 100: 364-370.

Barnard, J.L. \& J.D. Thomas. 1987b. A new species of Chevalia from the Caribbean Sea (Crustacea: Amphipoda). Proc. Biol. Soc. Wash. 100: 532-542.

Barnard, J.L. \& J.D. Thomas. 1987c. New species of Neomegamphopus from Tropical America (Crustacea: marine Amphipoda). Proc. Biol. Soc. Wash. 100: 147-163.

Barnard, J.L. \& J.D. Thomas. 1989a. Four species of Synopiidae from the Caribbean region (Crustacea: Amphipoda). Proc. Biol. Soc. Wash. 102: 362-374.

Barnard, J.L. \& J.D. Thomas. 1989b. A new species, Ampelisca burkei, (Crustacea: Amphipoda) from Florida. Proc. Biol. Soc. Wash. 102: 375-384.

Barnard, J.L. \& J.D. Thomas. 1990. Ensayara jumane, a new species from Belize, Caribbean Sea (Amphipoda, Lysianassidae). Proc. Biol. Soc.Wash. 103: 120-126.

Bellan-Santini, D. 1997. Amphipods of the cold deep community on the south Barbados. Crustaceana 70: $1-30$. 
Bellan-Santini, D. 1999. Ordre des Amphipodes (Amphipoda Latreille, 1816), p. 93-176. In P.P. Grassé (ed.). Trait de Zoologie. Anatomie, systématique, biologie. Tome VII, Fascicule IIIA. Crustacés Péracarides. Mémoires de l'Institut océanographique, Monaco, Francia.

Berge, J., W. Vader \& A. Galan. 2001. Type material of Stegocephalidae Dana, 1855 (Crustacea: Amphipoda) in the collections of The Natural History Museum, London, including the description of seven new species. Bull. Nat. Hist. Mus. Lond. (Zool.) 67: 109-136.

Blanco, J.P. 1980. Contribución al conocimiento de la biología de Talorchestia margaritae Stephensen 1948 (Amphipoda: Talitridae). Tesis de Licenciatura, Universidad de Oriente, Venezuela.

Bousfield, E.L. 1965. Haustoriidae of New England (Crustacea: Amphipoda). Proc. U.S. Nat. Mus. 117: 159-239.

Bousfield, E.L. 1969. New records of Gammarus (Crustacea: Amphipoda) from the middle Atlantic region. Chesapeake Science 10: 1-17.

Bousfield, E.L. 1970. Adoptive radiation of sand burrowing amphipod crustaceans. Chesapeake Science 11: 143154.

Bousfield, E.L. 1971. Amphipods of the Bismark Archipielago and adjacent Indo-Pacific islands. Steenstrupia 1: 255-293.

Bousfield, E.L. 1973. Shallow-water gammaridean amphipoda of New England. Comstock Publishing Associates, Cornell, Ithaca \& London, EEUU.

Bousfield, E.L. 1984. Recent advances in the systematics and biogeography of landhoppers (Amphipoda: Talitridae) of the Indo-Pacific Region. In F.J. Radovsky, P.H. Raven \& S.H. Sohmer (eds.). Biogeography of the Tropical Pacific. Bishop Mus. Spec. Publ. 72: 171-210.

Bousfield, E.L. 1991. New sandhoppers (Crustacea: Amphipoda) from the Gulf coast of the United States. Gulf Res. Rep. 8: 271-283.

Bousfield, E.L. \& P.M. Hoover. 1997. The amphipod superfamily corophioidea on the Pacific coast of north America. Part V. Family Corophiidae: Corophiinae, new subfamily. Systematics and distributional ecology. Amphipacifica 2: 67-139.

Bowers, D.E. 1964. Natural history of two beach hoppers of the genus Orchestoidea (Crustacea: Amphipoda) with reference to their complemental distribution. Ecology 44: 677-696.

Bynum, K.H. \& R.S. Fox. 1977. New and noteworthy amphipod crustaceans from north Carolina, U.S.A. Chesapeake Science 18: 1-33.

Camp, D.K., N.H. Whiting \& R.E. Martin. 1977. Nearshore marine ecology at Hutchinson Island, Florida: 19711974. V. Arthropods. Florida Mar. Res. Publications 25: 1-63.

Camp, D.K., W.G. Lyons \& T.H. Perkins. 1998. Checklists of selected shallow-water marine invertebrates of Florida. Florida Marine Research Institute Technical Report TR-3, Florida, EEUU.

Chevreux, E. \& L. Fage. 1925. Faune de France. Amphipodes 9: 1-488.

Christmas, J.Y. \& W. Langley. 1973. Estuarine invertebrates, Mississippi. p. 255-319. In J.Y. Christmas (ed.). Cooperative Gulf of Mexico Estuarine Inventory and Study, Mississippi. Mississippi Gulf Coast Research Laboratory, Mississippi, EEUU.

Ciavatti, G. 1989. Les talitrides (Crustacea, Amphipoda) des plages de la Guadeloupe, description de deux especes nouvelles. Ann. Inst. Oceanogr., Paris 65: 127-146.

Clark, J. \& J.L. Barnard. 1985. Lucayarina catacumba new genus, new species, a Bahamian sea-cave amphipod (Crustacea, Amphipoda, Lysianassidae). Proc. Biol. Soc. Wash. 98: 243-254.

Cloudsley-Thompson, J.L. 1988. Evolution and adaptation of terrestrial Arthropods. Springer-Verlag, Berlin, Alemania.

Conlan, K.E. 1995. Thumbing doesn't always make the genus: Revision of Microjassa Stebbing (Crustacea: Amphipoda: Ischyroceridae). Bull. Mar. Sci. 57: 333-377.

De la Fuente, J.A. 1994. Zoología de Artrópodos. Interamericana, McGraw-Hill, España.

Díaz, Y.J. 2001. Contribución al conocimiento del Orden Amphipoda de la costa Centro-Occidental de Venezuela. Tesis de Maestría, Universidad Simón Bolívar, Caracas. 542 p.

Díaz, Y.J. \& A. Martín. 2000a. Audulla chelifera (Amphipoda: Corophiidae) en las costas de Venezuela. Rev. Biol. Trop. 48: 722.

Díaz, Y.J. \& A. Martín. 2000b. Nasageneia yucatanensis (Amphipoda: Eusiridae) en las costas de Venezuela. Rev. Biol. Trop. 48: 724. 
Díaz, Y.J. \& A. Martín. 2000c. Eusiroides yucatanensis (Amphipoda: Eusiridae) en las costas de Venezuela. Rev. Biol. Trop. 49: 767.

Díaz, Y.J. \& A. Martín. 2000d. Listriella carinata (Amphipoda: Liljeborgiidae) en las costas de Venezuela. Rev. Biol. Trop. 49: 767.

Díaz, Y.J. \& A. Martín. 2001a. Amphilochus casahoya (Amphipoda: Amphilochidae) en las costas de Venezuela. Rev. Biol. Trop. 49: 1269.

Díaz, Y.J. \& A. Martín. 2001b. New records of amphipods (Crustacea: Amphipoda) from shallow water on the Caribbean coast of Venezuela. Rev. Biol. Trop. 49: $1271-1276$

Escobar-Briones, E. \& I. Winfield. 2003. Checklist of the benthic gammaridea and caprellidea (Crustacea: Peracarida: Amphipoda) from the Gulf of Mexico continental shelf and slope. Belg. J. Zool. 133: $37-44$.

Escobar-Briones, E., I. Winfield, M. Ortíz, E. Gasca \& E. Suárez. 2002. Amphipoda, p. 341-371. In J. Llorente \& J.J. Morrone (eds.). Biodiversidad, taxonomía y biogeografía de artrópodos de México: Hacia una síntesis de su conocimiento. Comisión Nacional para el Conocimiento y uso de la Biodiversidad. UNAM, México.

Farrell, D.H. 1970. Ecology and seasonal abundance of littoral amphipods from Mississippi. Tesis de Maestría, Mississippi State Univ., Mississippi, EEUU.

Foster, J.M. 1989. Acanthohaustorius uncinus, a new species of sand-burrowing amphipod from the northern Gulf of Mexico, with notes on its ecology (Haustoriidae: Haustoriinae). Gulf Res. Rep. 8: 189197.

Foster, J.M. \& S.E. LeCroy. 1991. Haustorius hayneae, a new species of haustoriid amphipod from the northern Gulf of Mexico, with notes on its ecology at Panama city, Florida U.S.A. Gulf Res. Rep. 8: 259-270.

Fox, R.S. 1973. Ceradocus shoemakeri and Eriopisa schoenerae, new amphipods (Crustacea: Gammaridae) from the Bahama Islands. J. Elisha Mitchell Sci. Soc. 89: 147-159.

Fox, R.S. \& K.H. Bynum. 1975. The amphipod crustaceans of North Carolina estuarine waters. Chesapeake Science 16: 223-237.

Gable, M.F. \& E.A. Lazo-Wasem. 1990. Lysianassidae (Amphipoda: Lysianassoidea) of Bermuda. J. Crust. Biol. 10: 721-734.
Galan, A. 1983. Systematic studies on Ericthonius brasiliensis (Crustacea, Amphipoda, Ischiroceridae) from the Caribbean Sea of Venezuela. Bol. Inst. Oceanogr. Univ. Oriente 22: 65-69.

Galan, A. 1984. A systematic study of Amphipoda (Crustacea) of the Caribbean coast of Venezuela. Tesis de doctorado, London University, Inglaterra.

Goeke, G.D. \& J.M. Gathof. 1983. Amphipods of the family Ampeliscidae (Gammaridea). II. Notes on the ocurrence of Ampelisca holmesi in the northern Gulf of Mexico. Gulf Res. Rep. 7: 289-291.

Goeke, G.D. \& R.W. Heard Jr. 1983. Amphipods of the family Ampeliscidae (Gammaridea) I. Ampelisca bicarinata, a new species of amphipod from the Gulf of Mexico. Gulf Res. Rep. 7: 217-223.

Goeke, G.D. \& R.W. Heard Jr. 1984. Amphipods of the family Ampeliscidae (Gammaridea) III. Ampelisca parapacifica, a new species of amphipod from the western North Atlantic with designation of a substitute name for A. eschrichtii pacifica Gurjanova, 1955. Gulf Res. Rep. 7: 331-337.

Hayward, P.J. \& J.S. Ryland. 1990. The marine fauna of the British Isles and North-West Europe. Introduction and Protozoans to Arthropods. Clarendon, Oxford, Inglaterra.

Hayward, P.J. \& J.S. Ryland. 1995. Handbook of the marine fauna of North-West Europe. Oxford University, Oxford, Inglaterra.

Heard, R.W. \& D.G. Perlmutter. 1977. Description of Colomastix janicese n. sp., a commensal amphipod (Gammaridea, Colomastigidae) from the Florida keys U.S.A. Proc. Biol. Soc. Wash. 90: 30-42.

Hedgpeth, J.W. 1954. Botton communities of the Gulf of Mexico. In Gulf of Mexico, its origin, waters, and marine life. Fish. Bull. 89: 203-214.

Hendrickx, M.E. 1995. Checklist of lobster-like decapod crustaceans (Crustacea: Decapoda: Thalassinidea, Astacidea and Palinura) from the eastern tropical Pacific. Anales Inst. Biol. Univ. Nac. Autón. México, Ser. Zool. 66: 151-163

Holsinger, J.R. 1974. Systematics of the subterranean amphipod genus Stygobromus (Gammaridae), Part I: Species of the western United States. Smith. Contrib. Zool. 160: 1-63.

Holsinger, J.R. 1990. Tuluweckelia cernua, a new genus and species of stygobiont amphipod crustacean (Hadziidae) from anchialine caves on the Yucatan Peninsula in Mexico. Beaufortia 41: 97-107. 
Holsinger, J.R. 1992. Two new species of the subterranean Amphipod genus Bahadzia (Hadziidae) from the Yucatan Peninsula region of southern Mexico, with an analysis of phylogeny and biogeography of the genus. Stygologia 7: 85-105.

Holsinger, J.R. \& J. Yager. 1985. A new genus and two new species of subterranean amphipod crustaceans (Hadziidae) from the Bahama Turks and Caicos Islands. Bijdr. Dierkd. 55: 283-294.

Holsinger, J.R. \& S.B. Peck. 1968. A new genus and species of subterranean amphipod (Gammaridea) from Puerto Rico, with notes on its ecology, evolution and relationship to other Caribbean amphipods. Crustaceana 15: 249-262.

Jaume, D. \& H.P. Wagner. 1998. New cave-dwelling amphipods (Lysianassidae, Hadziidae) from the Dominican Republic (Hispaniola). Smith. Contrib. Zool. 68: 37-66.

Just, J. 1977. Amphyllodomus incurvaria gen. nt. sp. n. (Crustacea: Amphipoda) a remarkable leaf-cutting amphitoid from marine shallows of Barbados. Zool. Scripta 6: 229-232.

Just, J. 1981. Tiron bellairsi sp. n. (Amphipoda, Synopiidae) from coral sand in Barbados, with notes on behaviour. Zool. Scripta 10: 259-263.

Just, J. 1984. Siphonoecetinae (Crustacea, Amphipoda, Corophiidae) 2: Caribboecetes Just, 1983, with description of six new species. Steenstrupia 10: 37-64.

Karaman, G.S. 1980a. Revision of the genus Gitanopsis Sars 1895 with description of new genera Afrogitanopsis and Rostrogitanopsis n. gen. (fam. Amphilochidae). Contributions to the knowledge of the Amphipoda 104. Poljoprivreda I Sumarstro 26: 43-69.

Karaman, G.S. 1980b. Cocoharpinia illifei, new genus and species from Bermuda, with remarks to other genera and species (Fam. Phoxocephalidae). Contribution to the Knowledge of the Amphipoda 103. Studia Marina, Kotor 10: 149-175.

Karaman, G.S. 1980c. Revision of genus Idunella Sars with description of new species I. sketi n. sp. (Fam. Liljeborgiidae). Acta Adriat. 21: 409-435.

Karaman, G.S. 1981. Redescription of Melita planaterga Kunkel 1910 from Bermuda Islands with revision of genera Melita Leach and Abludomelita n. gen. Poljoprivreda I Sumarstvo, Titograd 27: 29-50.
Karaman, G.S. 1987. A new species of the genus Melita Leach (fam. Melitidae) from Bermuda and Fiji Islands. Bull. Mus. Hist. Nat. Belgrade 42: 19-35.

Krapp-Schickel, T. 1976. Die Gattung Stenothoe (Crustacea, Amphipoda) im Mittelmeer. Bijdr. Dierkd. 48: 1-15.

Krapp-Schickel, T. \& S. Ruffo. 2000. The Maera quadrimana-complex (Crustacea Amphipoda, Melitidae) demands a new concept: Quadrimaera n. gen. (with description of three new species from Western Atlantic). Boll. Mus. Civico Storia Natur. Verona 24: 193-214.

Krapp-Schickel, T. \& S. Ruffo. 2001. A new Maeracoota from the Caribbean sea: Maeracoota galani n. sp. (Crustacea Amphipoda, Melitidae). Boll. Mus. Civico Storia Natur. Verona 25: 3-8.

Kunkel, B.W. 1910. The Amphipoda of Bermuda. Trans. Connecticut Acad. Arts and Sci. 16: 1-116.

Lagarde, G. 1987. Anfípodos gammaridea del litoral de Golfo Triste y áreas adyacentes. Caribb. J. Sci. 23: 260-277.

Lazo-Wasem, E.A. \& M.F. Gable. 1987. A review of recently discovered specimens of Bermuda Amphipoda (Crustacea: Peracarida) described by B.W. Kunkel (1882-1969). Proc. Biol. Soc. Wash. 100: 321-336.

LeCroy, S.E. 1995. Amphipod Crustacea III. Family Colomastigidae. Memoirs of the Hourglass Cruises. Volume IX, Part II: 1-139.

Ledoyer, M. 1979. Expedition Rumphius II (1975) crustaces parasites, commensaux, etc. (Th. Monod et R. Serene, ed.) Crustaces amphipods gammariens. Bull. Mus. Natl. d'Histoire Nat. Paris, Series 4. 1: 137-181.

Ledoyer, M. 1986. Faune mobile des herbiers de phanérogames marines (Halodule et Thalassia) de la Laguna de Terminos (Mexique, Campeche). II. Les gammariens (Crustacea). Anales Inst. Ciencias Mar y Limnol., Univ. Nac. Autón. México 13: 171-200.

Lewis, S.M. \& B. Kensley. 1982. Notes on the ecology and behaviour of Pseudamphitoides incurvaria (Just) (Crustacea, Amphipoda, Ampithoidae). J. Nat. Hist. 16: 267-274.

Lowry, J.K. 1972. Taxonomy and distribution of Microprotopus along the east coast of the U.S. (Amphipoda: Isaeidae). Crustaceana 3: 277-286. 
Lowry, J.K. 1974. A new species of the amphipod Biancolina from the Sargasso Sea. T. Am. Microsc. Soc. 93: 71-78.

Lowry, J.K. \& H.E. Stoddart. 1984. Redescription of Schellenberg's types of Lysianopsis subantactica and Paralysianopsis odhneri (Amphipoda, Lysianassidae). Crustaceana 47: 98-108.

Lowry, J.K. \& H.E. Stoddart. 1997. Amphipoda Crustacea IV. Families Aristiidae, Cyphocarididae, Endevouridae, Lysianassidae, Scopelocheiridae, Uristidae. Memoirs of the Hourglass Cruises. Florida Marine Research Institute, Volume X. Part I, Florida, EEUU.

Lowry, J.K. \& J.D. Thomas. 1991. A new species of Cerapus from Cudjoe Channel, lower Florida keys, USA, with notes on male behaviour (Crustacea: Amphipoda: Corophioidea). J. Nat. Hist. 25: 14611467.

Margulis, R.J. 1967. Deep-sea Ampeliscidae (Amphipoda, Gammaridea) from the Pacific Ocean. Crustaceana 13: 299-309.

Martín, A. 2001a. Westwoodilla longimana (Amphipoda: Oedicerotidae) en las costas de Venezuela. Rev. Biol. Trop. 49: 1270.

Martín, A. 2001b. Seba tropica (Amphipoda: Sebidae) en las costas de Venezuela. Rev. Biol. Trop. 49: 1270.

Martín, A., M. Ortíz \& D. Atienza. 2000. Una nueva especie de anfípodo del género Listriella (Crustacea, Amphipoda, Liljeborgiidae) de Venezuela. Bol. Centro Invest. Biol., Universidad del Zulia 34: 399-409.

Martín, A., M. Ortíz \& D. Atienza. 2001. Una nueva especie de anfípodo del género Amphilochus (Crustacea, Amphipoda, Amphilochidae) de Venezuela. Acta Científica Venezolana 52: 34-39.

Martín, A. \& Y.J. Díaz. 2003. La fauna de anfípodos (Crustacea: Amphipoda) de las aguas costeras de la región oriental de Venezuela. Bol. Instituto Español de Oceanografía 19: 327-344.

Martin, J.W. \& G.E. Davis. 2001. An update classification of the recent Crustacea. Natural History Museum of Los Angeles County. Science Series 39: 1-124.

Mateus, A. \& E. de O. Mateus. 1966. Amphipodes littoraux de Principe et de Sao Tome. Annales de L'Institut Oceanographique, Paris 44: 173-198.
McKinney, L.D. 1978. Amphilochidae (Crustacea: Amphipoda) from the western Gulf of Mexico and Caribbean Sea. Gulf Res. Rep. 6: 137-143.

McKinney, L.D. 1979. Liljeborgiid amphipods from the Gulf of Mexico and Caribbean Sea. Bull. Mar. Sci. 29: 140-154.

McKinney, L.D. 1980a. The genus Photis (Crustacea: Amphipoda) from the Texas coast with the description of a new species, Photis melanicus. Contrib. Mar. Sci. 23: 57-61.

McKinney, L.D. 1980b. Four new and unusual amphipods from the Gulf of Mexico and Caribbean Sea. Proc. Biol. Soc. Wash. 93: 83-103.

McKinney, L.D., R.D. Kalke \& J.S. Holland. 1978. New species of amphipods from the western Gulf of Mexico. Contrib. Mar. Sci. 21: 133-159.

Meglitsch, P.A. \& F.R. Schram. 1991. Invertebrate Zoology. Oxford University, Oxford, Inglaterra.

Mills, E.L. 1964. Ampelisca abdita, a new amphipod crustacean from eastern North America. Canadian J. of Zool. 42: 559-575.

Mills, E.L. 1965. The zoogeography of the north Atlantic and north Pacific ampeliscid Amphipoda crustaceans. Syst. Zool. 14: 119-130.

Mills, E.L. 1967. A reexamination of some species of Ampelisca (Crustacea: Amphipoda) from the east coast of north America. Canadian J. of Zool. 45: 635-652.

Mills, E.L. 1971. Deep-sea amphipoda from the western north atlantic ocean. The family Ampeliscidae. Limnol. Oceanogr. 16: 357-386.

Myers,A.A. 1968a. TwoAoridae(Amphipoda, Gammaridea) including a new species of Amphideutopus Barnard from Venezuelan waters. Crustaceana 14: 127-130.

Myers, A.A. 1968b. A new genus and two new species of gammaridean Amphipoda from Central America. J. Linn. Soc. (Zool.) 47: 527-531.

Myers, A.A. 1970. Taxonomic studies on the genus Grandidierella Coutiere (Crustacea, Amphipoda) with a description of $G$. dentimera sp. nov. Bull. Mar. Sci. 20: 135-147.

Myers, A.A. 1977a. Studies on the genus Lembos Bate. VI. Atlantic species: L. dentischium sp. nov., L. kunkelae 
sp. nov., L. rectangulatus sp. nov., L. unicornis Bynum \& Fox. Boll. Mus. Civ. St. Nat. Verona IV: 125-154.

Myers, A.A. 1977b. Studies on the genus Lembos Bate. V. Atlantic species: L. smithi (Holmes), L. brunneomaculatus sp. nov., L. minimus sp. nov., L. unifasciatus sp. nov. Boll. Mus. Civ. St. Nat. Verona IV: 95-124.

Myers, A.A. 1978. Studies on the genus Lembos Bate. VII Boll. Mus. Civ. St. Nat. Verona V: 183-209.

Myers, A.A. 1979. Studies on the genus Lembos Bate. VIII. Atlantic species. L. tigrinus sp. nov., L. tempus sp. nov., L. spinicarpus (Pearse) comb. nov. with spp inermis nov., L. ovalipes sp. nov., L. unifasciatus Myers ssp reductus nov. Boll. Mus. Civ. St. Nat. Verona VI: 221-248.

Myers, A.A. 1981. Amphipoda Crustacea. I. Familia Aoridae. Memoirs of the Hourglass Cruises 5: 1-75.

Nelson, W.G. \& L. Demetriales. 1992. Peracarids associated with sabellarid worm rock (Phragmatopoma lapidosa Kinberg) at Sebastian inlet, Florida, U.S.A. J. Crustacean Biol. 12: 647-654.

Odum, W.E. 1975. Pathways of energy flow in a south Florida estuary. Tesis de doctorado, University of Miami, EEUU.

Ortíz, M. 1976a. Un nuevo anfípodo perforador de madera (Amphipoda, Gammaridea, Cheluridae) de aguas cubanas. Invest. Mar. Serie 8: 21-26.

Ortíz, M. 1976b. Un nuevo anfípodo de aguas cubanas (Amphipoda, Gammaridea, Phliantidae). Invest. Mar. Serie 8: 21-35.

Ortíz, M. 1976c. A new crustacean amphipod, Mallacoota carausui, from the cuban waters. Revue Roumaine de Biologie 21: 93-95.

Ortíz, M. 1976d. Un nuevo género y una nueva especie de anfípodo de aguas cubanas (Amphipoda, Gammaridea, Ampithoidae). Invest. Mar. Serie 8: 3-12.

Ortíz, M. 1976e. Un nuevo anfípodo (Amphipoda, Gammaridea, Gammaridea) colectado del contenido estomacal del ronco amarillo Haemulun sciurus, de aguas cubanas. Invest. Mar. Serie 8: 13-20.

Ortíz, M. 1978. Invertebrados marinos bentósicos de Cuba. I. Crustacea Amphipoda. Gammaridea. Invest. Mar. Serie 8. 38: 3-10.
Ortíz, M. 1979a. Lista de especies y bibliografía de los anfípodos (Crustacea: Amphipoda) del Mediterráneo americano. Invest. Mar. Serie 8: 1-40.

Ortíz, M. 1979b. Contribución al estudio de los anfípodos (Gammaridea) del mediterráneo americano. Invest. Mar. Serie 8: 1-16.

Ortíz, M. 1980. Una nueva especie de anfípodo bentósico (Amphipoda, Gammaridea) de aguas cubanas. Rev. Invest. Mar. 1: 92-103.

Ortíz, M. 1983. Los anfípodos (Gammaridea) de las costas del Mar Caribe de la República de Colombia. Rev. Invest. Mar. 4: 23-31.

Ortíz, M. 1991. Amphipod Crustacea. II. Family Bateidae. Memoirs of the Hourglass Cruises VII: 1-31.

Ortíz, M., S. Chazaro-Olvera \& I. Winfield. 2001. A new amphipod crustacean of the genus Haustorius (Gammaridea, Haustoriidae), from the east coast of México. Avicennia 14: 53-59.

Ortíz, M. \& O. Gómez. 1979. Una nueva especie de anfípodo (Amphipoda, Gammaridea) de aguas profundas del sur de Cuba. Invest. Mar. Serie 8. 40: 23-30.

Ortíz, M. \& R. Lalana. 1980. Un nuevo anfípodo del género Leptocheirus (Amphipoda, Gammaridea) de aguas cubanas. Rev. Invest. Mar. 1: 58-73.

Ortíz, M. \& R. Lalana. 1989. Una nueva especie de anfípodo del complejo Eriopisa (Amphipoda, Gammaridea), de aguas cubanas. Rev. Invest. Mar. 10: 233-237.

Ortíz, M. \& R. Lalana. 1993. Adición a la lista de especies y bibliografia de los anfipodos (Crustacea, Amphipoda) del Mediterráneo Americano. Rev. Invest. Mar. 14: 16-37.

Ortíz, M. \& R. Lalana. 1994a. A new amphipod species of the genus Nasageneia (Gammaridea: Eusiridae) from the south-western cuban marine waters. Travaux du Muséum d'Histoire naturelle Grigore Antipa 34: 285-292.

Ortíz, M. \& R. Lalana. 1994b. Two new species of the genus Elasmopus (Amphipoda, Gammaridea), from the Cuban marine waters. Travaux du Muséum d'Histoire naturelle Grigore Antipa 34: 293-302.

Ortíz, M. \& R. Lalana. 1995. Gitanopsis templadoi, una nueva especie de anfípodo (Gammaridea, Amphilochidae), de la plataforma suroccidental de Cuba. Anales Inst. Cien. Mar y Limnol, Univ. Nac. Autón. México 22: 121-124. 
Ortíz, M. \& R. Lalana. 1996. Los anfípodos de la primera expedición conjunta Cuba-US, a bordo del B/I "Ulises", a las aguas del Archipiélago SabanaCamagüey, Cuba, en 1994. Anales Inst. Biol. Univ. Nac. Autón. México Ser. Zool. 67: 89-101.

Ortíz, M. \& R. Lalana. 1998. Lista actualizada de los crustáceos no decápodos de Cuba. Rev. Invest. Mar. 19: 92-99.

Ortíz, M. \& R. Lalana. 2002. Primer registro del género Neoischyrocerus (Amphipoda, Ischyroceridae) para el Mar Caribe y el Archipiélago cubano, con la descripción de nueva especie de Cuba. Avicennia 15: $37-51$.

Ortíz, M., R. Lalana \& J. Beltran. 1993. Una nueva especie de anfípodo hadzioideo (Amphipoda: Gammaridea) del Caribe de Nicaragua. Rev. Invest. Mar. 14: $103-$ 109.

Ortíz, M., R. Lalana \& V. Lio. 1999. Un nuevo género y una especie de anfípodo marino (Amphipoda: Aristiidae), de Cuba. Avicennia 10/11: 137-142.

Ortíz, M., R. Lalana \& V. Lio. 2001. Primera consignación del género Protohadzia (Amphipoda, Gammaridea), para el archipiélago cubano. Avicennia 14: 129-132.

Ortíz, M., R. Lalana \& M. López. 1992. Nueva especie de anfípodo del género Bemlos (Gammaridea, Corophidae, Corophidae), de Cuba. Anales Inst. Cienc. Mar y Limnol. Univ. Nac. Autón. México 19: 163-166.

Ortíz, M., R. Lalana \& A. Sánchez-Díaz. 2000b. Una nueva especie de anfípodo espongícola del género Hoplopheonoides Shoemaker, 1956 (Gammaridea, Cyproideidae) de Cuba. Avicennia 12/13: 63-68.

Ortíz, M. \& R. Lemaitre. 1994. Crustáceos anfípodos (Gammaridea) colectados en las costas del Caribe colombiano, al sur de Cartagena. An. Inst. Invest. Mar. Punta Betin 23: 119-127.

Ortíz, M. \& R. Lemaitre. 1997. Seven new amphipods (Crustacea, Peracarida, Gammaridea) from the Caribbean coast of South America. Bol. Invest. Mar. Cost. 26: 71-104.

Ortíz, M., A. Martín \& D. Atienza. 2000a. Una nueva especie de anfípodo del género Tiburonella (Crustacea, Amphipoda, Platyischnopidae) de Venezuela. Acta Biol. Venez. 20: 29-36.
Ortíz, M. \& J. Nazábal. 1984a. A new amphipod crustacean of the genus Lembos (Gammaridea, Aoridae), from the cuban marine waters. Travaux du Museum d'Historie naturelle Grigore Antipa XXVI: 11-13.

Ortíz, M. \& J. Nazábal. 1984b. Corocubanus, un nuevo género de anfipodo (Amphipoda Gammaridea, Corophiidae), de aguas cubanas. Rev. Invest. Mar. 5: 3-21.

Ortíz, M. \& J. Nazábal. 1988. Una nueva especie de anfípodo del género Lembos (Crustacea, Amphipoda), de aguas cubanas. Rev. Invest. Mar. 9: 29-35.

Ortíz, M. \& A. Pérez. 1995. Una nueva especie de anfípodo cavernícola Hadzioideo (Amphipoda, Gammaridea) de Cuba. Graellsia 51: 165-168.

Ortíz, M. \& T. Veledo. 1985. Una nueva especie de anfípodo del género Garosyrrhoe (Synopiidae, Gammaridea) de aguas cubanas. Rev. Invest. Mar. 6: 14-18.

Ortíz, M. \& I. Winfield. 1995. Anfípodos (Crustacea: Gammaridea) asociados a Ruppia maritima. Nota sobre su distribución geográfica. Rev. Zool. 7: 1-5.

Pearse, A.S. 1908. Descriptions of four new species of amphipodous Crustacea from the Gulf of Mexico. Proc. U.S. Nat. Mus. 34: 27-32.

Pearse, A.S. 1913. Notes on certain Amphipoda from the Gulf of Mexico with descriptions of new genera and new species. Proc. U.S. Nat. Mus. 43: 369-379.

Platvoet, D. 1983. Bogidiella (B.) neotropica Ruffo, 1952 (Crustacea, Amphipoda) rediscovery in Venezuela. Bijdr. Dierkd. 53: 109-114.

Robertson, P.B. \& C.R. Shelton. 1978. Two new species of haustoriid amphipods (Crustacea: Amphipoda) from the northwestern Gulf of Mexico. Smith. Contrib. Mar. Sci. 21: 47-62.

Robertson, P.B. \& C.R. Shelton. 1980. Lepidactylus triarticulatus n. sp., a new haustoriid amphipod from the northern Gulf of Mexico. Gulf Research Reports 6: 415-420.

Rodríguez, G. 2000. El Sistema de Maracaibo. Biología y Ambiente. Instituto Venezolano de Investigaciones Científicas (IVIC), Caracas, Venezuela.

Ruffo, S. 1950. Studi sui crostacei anfipodi. XXII. Anfipodi del Venezuela raccolti dal dott. G. Marcuzzi. Mem. Mus. Civico Storia Natur. Verona 2: 49-65. 
Ruffo, S. 1954. Studi sui crostacei anfipodi. XL. Nuovi anfipodi raccolti nel Venezuelan dal Prof. G. Marcuzzi. Mem. Mus. Civico Storia Natur. Verona 4: 117- 125.

Ruffo, S. 1957. Una nuova specie troglobia di Hyalella del Venezuela (Amphipoda, Talitridae): viaggi in Venezuela di Nino Sanfilippo, IV. Ann. Mus. Civico Storia Natur. Genova 69: 363-269.

Ruffo, S., T. Krapp-Schickel \& M.F. Gable. 2000. The genus Maera (Crustacea: Amphipoda: Melitidae) from Bermuda. Postilla 221: 1-35.

Sheridan, P.F. 1979. Three new species of Melita (Crustacea, Amphipoda), with notes on the amphipod fauna of the Apalachicola estuary of northwest Florida. Northeast Gulf Sci. 3: 60-73.

Shoemaker, C.R. 1921. Report on the amphipods collected by the Barbados-Antigua expedition from the University of Iowa. Stud. Nat. History 9: 99-102.

Shoemaker, C.R. 1926. The amphipods of the family Bateidae in the collection of the United States National Museum. Proc. U.S. Nat. Mus. 68: 1-26.

Shoemaker, C.R. 1930. The amphipoda of the Chetincamp expedition of 1917. Contrib. Can. Biol. Fish. 5: 221-359.

Shoemaker, C.R. 1932. The amphipod Nototropis minikoi on the east coast of the United States. Proc. Biol. Soc. Wash. 45: 199-200.

Shoemaker, C.R. 1933a. Two new genera and six new species of Amphipoda from Tortugas. Papers of the Tortugas Laboratory 28: 245-256.

Shoemaker, C.R. 1933b. Amphipoda from Florida ans the West Indies. American Mus. Novit. 598: 1-24.

Shoemaker, C.R. 1934. Three new amphipods. Reports on collections obtained by the First Johnson-Smithsonian deep-sea expedition to the Puerto Rican deep. Smith. Misc. Collect. 91: 1-6.

Shoemaker, C.R. 1935. The amphipods of Porto Rico and Virgin Islands. Trans. N.Y. Acad. Sci. 15: 229-253.

Shoemaker, C.R. 1942. Amphipod crustaceans collected on the Presidential Cruise of 1938. Smith. Misc. Collec. 101: $1-52$.

Shoemaker, C.R. 1943. A new amphipod of the genus Corophium from Florida. The Charleston Museum Leaflet 18: 1-7.
Shoemaker, C.R. 1945a. The Amphipod genus Photis on the east coast of North America. The Charleston Museum Leaflet 22: 1-17.

Shoemaker, C.R. 1945b. The amphipod genus Unciola on the east coast of America. The Am. Midland Nat. 34: 446-465.

Shoemaker, C.R. 1945c. The amphipoda of the Bermuda Oceanographic Expeditions 1929-1931. Zoologica 30: 185-266.

Shoemaker, C.R. 1947. Further notes on the amphipod genus Corophium from the east coast of America. J. Wash. Acad. Sci. 37: 47-63.

Shoemaker, C.R. 1948. The Amphipoda of the Smithsonian Roebling Expedition to Cuba in 1937. Smith. Misc. Collec. 110: 1-15.

Shoemaker, C.R. 1956. Observations on the amphipod genus Parhyale. Proc. U.S. Nat. Mus. 106: 345-358.

Shoemaker, C.R. 1959. Three new cave amphipods from the West Indies. J. Wash. Acad. Sci. 49: 273-283.

Smith, D.G. \& R.W Heard. 2001. A new species of Chelorchestia (Amphipoda: Talitridae) from southwest Florida, with a comments on the other species within the genus. J. of Crustacean Biol. 21: 1031-1041.

Springer, H. \& H.R. Bullis. 1956. Collections by the Oregon in the Gulf of Mexico. List of Crustaceans, Mollusks, and Fishes identified from collections made by the exploratory fishing vessel Oregon in the Gulf of Mexico and adjacent seas 1950 through 1955. Spec. Sci. 196: 1-134.

Stebbing, T.R.R. 1903. Amphipoda from Costa Rica. Proc. U.S. Nat. Mus. 26: 925-930.

Stebbing, T.R.R. 1906. Amphipoda I. Gammaridae. Das Tierriech 21: 1-806.

Stebbing, T.R.R. 1888. Report on the Amphipoda collected by H.M.S. Challenger during the years 1873-1876. In Great Britain Rept. Sci. Res. Voy. H.M.S. Challenger durin the years 1873-76. Zoology 29: 1-1 737.

Stebbing, T.R.R. 1897. Amphipods from the Conpenhagen Museum and other sources. Trans. Limnol. Soc. London (serie 2, Zool) 7: 25-45.

Steel, P. \& S.B. Collard. 1981. First Gulf of Mexico record for Biancolina brassiacephala (Amphipoda Biancolinidae). Northeast Gulf Sci. 4: 115-118. 
Stephensen, K. 1933a. Fresh- and brackish-water Amphipoda from Bonaire, Curaçao und Aruba. (Zoologische Ergebnisse einer Reise nach Bonaire, Curaçao, und Aruba im Jahre 1930). Zool. Jahrb. (Syst.) 64: 414-436.

Stephensen, K. 1933b. Amphipoda from the marine salines of Bonaire and Curaçao. (Zoologische Ergebnisse einer Reise nach Bonaire, Curaçao und Aruba im Jahre 1930). Zool. Jahrb. (Syst.) 64: 437-446.

Stephensen, K. 1947. Amphipods from Curaçao, Bonaire, Aruba and Margarita. Studies on the Fauna of Curacao, Aruba, Bonaire and the Venezuelan Islands 11: $1-20$

Stock, J.H. 1977. The taxonomy and zoogeography of the Hadziid amphipoda, with emphasis on the West Indian taxa. Studies on the Fauna of Curacao and other Caribbean Islands 177: 1-130.

Stock, J.H. 1980. A new cave amphipod (Crustacea) from Curacao: Psammogammarus caesicolus n. sp. Bijdr. Dierkd. 50: 375-386.

Stock, J.H. 1981. The taxonomy and zoogeography of the family Bogidiellidae (Crustacea, Amphipoda), with emphasis on the west Indian taxa. Bijdr. Dierkd. 51: 345-374.

Stock, J.H. 1983a. A new species of Psammogammarus (Crustacea, Amphipoda) from the Roques Archipielago, Venezuela. Bijdr. Dierkd. 53: 103-108.

Stock, J.H. 1983b. The stygobiont amphipoda of Jamaica. Bijdr. Dierkd. 53: 267-286.

Stock, J.H. 1985. Bogidiellidae (Amphipoda) from Haiti and some general rules on the occurrence of Crustacea Malacostraca in inland groundwaters of the West Indies. Stygologia 1: 208-223.

Stock, J.H. 1986. Two new amphipod crustaceans of the genus Bahadzia from 'blue holes' in the Bahamas and some remarks on the origin of the insular stygofaunas of the Atlantic. J. Nat. Hist. 20: 921-933.

Stock, J.H. \& L. Botosaneanu. 1983. Premiere decouverte d'amphipodes Gammaridea du groupement des Hadziides dans des eaux souterraines de l'Amerique du Sud: description de Metaniphargus venezolanus sp. n. Bijdr. Dierkd. 53: 158-164.

Stock, J.H., B. Sket \& T.M. Iliffe. 1987. The status of Bogidiella balearica Dancau, 1973, a stygobiont amphipod from Mallorca. Endins 13: 39-46.
Stock, J.H. \& J.J. Vermeulen. 1982. A representative of the mainly abyssal family Pardaliscidae (Crustacea, Amphipoda) in cave waters of the Caicos Islands. Bijdr. Dierkd. 52: 3-12.

Stoner, A.W. \& F.G. Lewis. 1985. The influence of quantitative and qualitative aspects of $t$ complexity in tropical sea-grass meadows. J. Exp. Mar. Biol. Ecol. 94: 19-40.

Thomas, J.D. 1976. A survey of primitive amphipods of Barataria Bay, Louisiana Region. Constrib. Mar. Sci. 20: $87-100$.

Thomas, J.D. 1979. A redescription of the wood-rasping amphipod Tropichelura gomezi Ortíz, 1976 (Cheluridae) from the Florida keys, with notes on its distribution and ecology. Proc. Biol. Wash. 92: 863-872.

Thomas, J.D. 1993. Identification Manual for the Marine Amphipoda (Gammaridea). I. Common Coral reef and rocky bottom amphipods of South Florida. Florida Department of Environment Proteccion, Florida, EEUU.

Thomas, J.D. 1997. Systematics, ecology and phylogeny of the Anamixidae (Crustacea: Amphipoda). Records of the Australian Museum 49: 35-98.

Thomas, J.D. \& J.L. Barnard. 1983. The Platyschnopidae of America (Crustacea: Amphipoda). Smith. Contrib. Zool. 375: 1-33.

Thomas, J.D. \& J.L. Barnard. 1984. Acanthohaustorius pansus, a new species of sand-burrowing amphipod from Looe Key reef, Florida keys, with redescription and distribution data of Acanthohaustorius bousfieldi Frame, 1980 (Amphipoda: Haustoriidae). Proc. Biol. Soc. Wash. 97: 909-926.

Thomas, J.D. \& J.L. Barnard. 1985a. Perioculodes cerasinus, n. sp., the first record of the genus from the Caribbean sea (Amphipoda: Oedicerotidae). Proc. Biol. Soc. Wash. 98: 98-106.

Thomas, J.D. \& J.L. Barnard. 1985b. A new marine genus of the Maera group (Crustacea: Amphipoda) from Belize. Proc. Biol. Soc. Wash. 98: 630-635.

Thomas, J.D. \& J.L. Barnard. 1986a. New genera and species of the Megaluropus group (Amphipoda, Megaluropidae) from American seas. Bull. Mar. Sci. 38: 442-476.

Thomas, J.D. \& J.L. Barnard. 1986b. Two species of Hornellia (subgenus Metaceradocus) from the Florida 
keys and Belize (Amphipoda, Melphidippiodea). Bull. Mar. Sci. 38: 477-487.

Thomas, J.D. \& J.L. Barnard. 1988. Elasmopus balkomanus, a new species from the Florida keys (Crustacea, Amphipoda). Proc. Biol. Wash. 101: 838-842.

Thomas, J.D. \& J.L. Barnard. 1989. Gammaropsis arawakia, a new species of marine amphipoda (Crustacea) from Jamaica. Proc. Biol. Wash. 102: 89-94.

Thomas, J.D. \& J.L. Barnard. 1990. Gitana dominica, a new species from the Caribbean Sea (Amphipoda: Amphilochidae). Proc. Biol. Wash. 103: 617-623.

Thomas, J.D. \& J.L. Barnard. 1991a. A review of the genus Iphimedia (crustacean: Amphipoda) with descriptions of three new species from Australia, Papua New Guinea and Florida. Invertebr. Taxon. 5: 469-485.

Thomas, J.D. \& J.L. Barnard. 1991b. Photis trapherus, a new elephantine species from the Florida Keys (Crustacea, Amphipoda). Proc. Biol. Wash. 104: 96-100.

Thomas, J.D. \& J.L. Barnard. 1992. Podocerus kleidus, new species from the Florida Keys (Crustacea, Amphipoda, Dulichiidae). Bull. Mar. Sci. 51: 309314.

Thomas, J.D. \& R.W. Heard. 1979. A new species of Cerapus Say, 1817 (Crustacea, Amphipoda) from the northern Gulf of Mexico, with notes on its ecology. Proc. Biol. Wash. 92: 98-105.

Thomas, J.D. \& M. Ortíz. 1995. Leucothoe laurensi, a new species of leucothoid amphipod from Cuban waters (Crustacea, Amphipoda, Leucothoidae). Proc. Biol. Soc. Wash. 108: 613-616.

Thomas, J.D. \& K.N. Klebba. 2006. Studies of commensal leucothoid amphipods: two new sponge-inhabiting species from South Florida and the Western Caribbean. J. Crust. Biol. 26: 13-22.

Van der Ham, J.L. \& R. Vonk. 2003. A phylogenetic analysis of the Eriopisa complex (Crustacea: Amphipoda: Melitidae) and a new species from beach interstitia in Venezuela. J. Nat. Hist. 37: 779-796.
Venables, B.J. 1981. Aspects of the population biology of a venezuelan beach amphipod, Talorchestia margaritae (Talitridae), including estimates of biomass and daily production and respiration rates. Crustaceana 41: 271-285.

Vonk, R. 1988. Psammomelita uncinata n.g., n. sp. (Crustacea, Amphipoda, Melitidae) from infralittoral sand interstices on Curaçao. Stygologia 4: 166-176.

Vonk, R. 1989. Nuuanu curvata $\mathrm{n}$. sp. and Melita leiotelson n. sp. (Crustacea, Amphipoda) from beach interstitial on Curaçao. Foundation for Scientific Research in Surinam and the Netherlands Antilles, Amsterdam 123: $185-198$.

Vonk, R. 1990. Thalassostygius exiguus n.g., n. sp., a new marine interstitial melitid (Crustacea, Amphipoda) from Curaçao and Klein Bonaire (Netherlands Antilles). Stygologia 5: 43-48.

Vonk, R.1991. Two marine interstitial Metaniphargus species (Crustacea, Amphipoda) from Hawaii and the Cayman Islands. Stygologia 6: 111-118.

Vonk, R. \& J.H. Stock. 1987. Psammogammarus longidactilus n. sp., a new cave amphipod (Crustacea) and other stygobiont amphipods from Bonaire. Stygologia 3: $241-251$.

Watling, L. 1981. Amphipoda from the northwestern Atlantic: the genera Jerbania, Epimeria, and Harpinia. Sarsia 66: 203-211.

Winfield, I. 1999. Peracáridos (Crustacea) asociados a praderas de pastos marinos (Ruppia maritima) del sistema lagunar de Alvarado, Veracruz: un análisis ecológico espacio-temporal. Tesis de Maestría, Universidad Nacional Autónoma de México, México D.F., México.

Winfield, I. \& M. Ortíz. 1996. Riqueza específica de crustáceos peracáridos en el Complejo Lagunar de Alvarado, Veracruz y sistemas circundantes. Rev. Zool. 2: 57-68.

Winfield, I., M. Ortíz, J. Franco \& C. Bedia. 1997. Distribución y diversidad del superorden Peracarida asociado a pastos marinos de Alvarado, Veracruz. Cuad. Mex. Zool. 3: 1-8. 